1 Title: Speciation of the Mycobacterium tuberculosis complex using mass spectrometry: a

2 proof-of-concept.

3 Running title: Speciation of mycobacteria by MALDI-TOF

4 Author: Simon Robinne ${ }^{1,2 \#}$, Jamal Saad ${ }^{1,3 \#}$, Madjid Morsli ${ }^{1,3}$, Hamidou Zelika Harouna ${ }^{1,3,4}$,

5 Fatah Tazerart $^{1,3,5}$, Michel Drancourt ${ }^{1,2,3}$, Sophie Alexandra Baron ${ }^{1,2,3^{*}}$

\title{
6 Affiliation:
}

7 1. Aix-Marseille-Univ., IRD, MEPHI, Marseille, France.

8 2. Assistance Publique-Hôpitaux de Marseille, France.

9 3. IHU Méditerranée Infection, Marseille, France.

10 4. Laboratoire National de Référence des IST/VIH et de la Tuberculose, Niamey, Niger.

11 5. Institut des Sciences Vétérinaires- Université de Blida 1, Blida- Algeria.

12 \# These two authors equally contributed to this work.

13 * Corresponding author: Sophie Alexandra Baron, Aix Marseille Univ, IRD, APHM,

14 MEPHI, IHU Méditerranée Infection, Faculté de Médecine et de Pharmacie, 19-21 boulevard

15 Jean Moulin, 13385 Marseille CEDEX 05, France. Phone: (33) 4137324 01. Email:

16 sophie.baron@ap-hm.fr

18 Word count abstract: $164<250$

19 Word count text: 2,367

20 Keywords: Mycobacterium, Mycobacterium tuberculosis, tuberculosis, MALDI-TOF mass

21 spectrometry, whole genome sequencing, identification. 


\section{ABSTRACT}

26 Mycobacteria that form the Mycobacterium tuberculosis complex are responsible for deadly

27 tuberculosis in animals and patients. Identification of these pathogens at the species level is

28 of primary importance for treatment and source tracing, and currently relies on DNA

29 analysis, including whole genome sequencing (WGS), which takes a whole day. In this study,

30 we report on the unprecedented identification of the $M$. tuberculosis complex species using

31 matrix-assisted laser desorption ionization-time of flight mass spectrometry (MALDI-TOF-

32 MS), with WGS as the comparative gold standard. In a first step, an optimised peptide

33 extraction applied to 24 isolates otherwise identified in three of the $11 \mathrm{M}$. tuberculosis

34 complex species by WGS, yielded 94 MALDI-TOF spectra which clustered according to

35 WGS identification. In a second step, 70/74 (95\%) other isolates were correctly identified at

36 the species level by this clustering method. This study is the first to report a MALDI-TOF-

37 MS method of identification of $M$. tuberculosis complex mycobacteria at the species level

38 and is easily implantable in clinical microbiology laboratories. 


\section{INTRODUCTION}

51 Mycobacteria that form the Mycobacterium tuberculosis complex are responsible for

52 tuberculosis, a deadly infection that is a major public health concern around the world (1).

53 The M. tuberculosis complex is composed of 11 different species: Mycobacterium

54 tuberculosis, Mycobacterium africanum, Mycobacterium orygis, Mycobacterium bovis,

55 Mycobacterium microti, Mycobacterium canettii, Mycobacterium caprae, Mycobacterium

56 pinnipedii, Mycobacterium suricattae, Mycobacterium dassie, and Mycobacterium mungi

57 (also related as ecotypes) (2). In our clinical microbiology practice however, M. tuberculosis

58 stricto sensu, along with M. bovis and its derivative, Bacillus Calmette-Guérin (BCG), and M.

59 africanum, are the sole $M$. tuberculosis complex species that we have encountered in 10

60 years.

61 The identification of M. tuberculosis isolates at the species level is a concern for the

62 immediate medical management of patients as, for example, $M$. bovis and BCG are two

63 species acknowledged to be pyrazinamide-resistant (3). It is also of concern in terms of

64 tracing the source in order to prevent additional cases, in the case of zoonotic M. bovis

65 tuberculosis for example (4).

66 While real-time PCR detection of the M. tuberculosis complex directly in clinical

67 samples is now widely used for the routine screening of tuberculosis, this technique does not

68 allow for a specific identification within the complex (5). In routine clinical microbiology,

69 the identification of the M. tuberculosis complex at the species level is achieved by DNA

70 sequence investigations and, at best, by whole genome sequencing (WGS) of the colony (5).

71 These performing techniques, however, require specific equipment that may not be available

72 at the point-of-care, with results taking a few days to become available (Saad J., Baron S.,

73 Amrane S., Gallou J., Brieu N. and Drancourt M., submitted for publication). 
75 TOF-MS), a widely implanted technique for the routine identification of bacteria in clinical

76 microbiology laboratories as a rapid and lower cost method for identifying bacterial species

$77(6,7)$, is also routinely used for the identification of mycobacteria $(8,9)$. However, MALDI-

78 TOF-MS suffers from the same limitation as real-time PCR, i.e., it is impossible to

79 differentiate $M$. tuberculosis complex species based on similar peptide profiles measurable

80 with currently available MALDI-TOF-MS protocols. The results is that it is currently

81 impossible to specify tuberculosis species based on this method (10).

In this study, we investigated whether clustering MALDI-TOF-MS spectra obtained

83 from M. tuberculosis complex colonies using principal component analysis (PCA) could

84 achieve species identification, by cross-referencing the data retrieved from genome

85 sequencing. PCA clustering has already proven to be a mathematical technique which can be

86 widely applied to MALDI-TOF-MS datasets, and is able to discriminate between groups of

87 isolates based on their peptide profile (11).

MATERIALS AND METHODS.

90 Mycobacterial isolates. A total of 105 clinical isolates cultured in the BSL3 laboratory of the

91 IHU Méditerranée Infection, Marseille, France (N=85) and the Laboratoire National de

92 Référence des IST/VIH et de la Tuberculose, Niamey, Niger $(\mathrm{N}=20)$ were included in this

93 study. This collection of isolates has previously been investigated, in part for accurate

94 identification by WGS, here used as the gold standard (Saad J., Baron S., Amrane S., Gallou

95 J., Brieu N. and Drancourt M, submitted for publication).

96 In a first phase, 24 isolates from the IHU Méditerranée Infection, identified at the species

97 level by WGS in the M. tuberculosis complex (M. tuberculosis, M. africanum, M. bovis),

98 were defined as reference strains for the construction of a standard PCA cluster of MALDI- 
99 TOF-MS spectra. These isolates were selected to be representative of the diversity of species

100 and genetic lineages encountered at the IHU Méditerannée Infection laboratory.

101 In a second phase, 74 other isolates from the IHU Méditerranée Infection (Marseille, France)

102 and the Laboratoire National de Référence des IST/VIH et de la Tuberculose (Niamey,

103 Niger), identified at the species level by WGS in the M. tuberculosis complex (M.

104 tuberculosis, M. africanum, M. bovis), were defined as our test group, in order to evaluate the

105 reliability of the identification of the standard cluster previously generated. Finally, seven

106 isolates from the IHU Méditerranée Infection, identified at the species level by WGS outside

107 the M. tuberculosis complex (two isolates of Mycobacterium abscessus, two Mycobacterium

108 avium, Mycobacterium chelonae, Mycobacterium gilvum, Mycobacterium persicum) were

109 used as negative controls of our PCA clustering method. All isolates were cultured on

110 TransBK m4 agar medium (Culture-Top, Les Ulys, France), a culture medium derived from

111 Middlebrook $7 \mathrm{H} 10$ agar medium at $37^{\circ} \mathrm{C}$ under a $5 \% \mathrm{CO} 2$ atmosphere for a minimum of

112 seven days. Total DNA extraction and WGS were performed as previously described (Saad

113 J., Baron S., Amrane S., Gallou J., Brieu N. and Drancourt M, submitted for publication).

\section{MALDI-TOF-MS analysis.}

115 Between one and three $1-\mu \mathrm{L}$ plastic loops harvested from each isolate were mixed with 300

$116 \mu \mathrm{L}$ of high purity liquid chromatography (HPLC)-grade water into a 1.5 -mL tube (Sarstedt,

117 Nümbrecht, Germany). Samples were then heated to $100^{\circ} \mathrm{C}$ for 30 minutes in order to

118 inactivate mycobacteria (12). After cooling, $900 \mu \mathrm{L}$ of ethanol was added, vortexed for one

119 minute and centrifuged for two minutes at 16,060xg, and the supernatant was eliminated. The

120 centrifugation step was repeated twice, and the pellet was dried at room temperature for

121 several minutes. A spatula-full of glass beads for cell disruption (diameter: $0.5 \mathrm{~mm}$; Scientific

122 Industries Inc., Bohemia, USA) and $20 \mu \mathrm{L}$ of acetonitrile were added, then samples were 
123 stirred using a vortex device for one minute. $20 \mu \mathrm{L}$ of a solution of $70 \%$ formic acid was then

124 added and agitated for five seconds. Samples were then agitated using a FastPrep-24TM

125 device (MP Biomedicals, \#6004500) for five cycles of 20 seconds, with a resting time of five

126 seconds between each cycle, then centrifuged for two minutes at 16,060xg. Four replicates of

$1271.5 \mu \mathrm{L}$ supernatant were deposited on a MALDI target plate (MSP 96 target polished steel,

128 Bruker Daltonics, Bremen, Germany) and left to dry at room temperature for one minute.

129 Matrix solution consisting of $1.5 \mu \mathrm{L}$ of a saturated $\square$-cyano 4-hydroxycinnamic acid (CHCA)

130 (Sigma-Aldrich, Taufkirchen, Germany) in TFA 5\% acetonitrile (1:1) was deposited on each

131 spot and left to dry for five minutes at room temperature. Two spots consisting of $1.5-\mu \mathrm{L}$

132 CHCA matrix only were incorporated as negative controls and two spots consisting of $1.5-\mu \mathrm{L}$

133 1/10 diluted solution of Bruker Bacterial Standard Test, an Escherichia coli extract spiked

134 with two high molecular weight proteins (Bruker Daltonics), mixed with 1.5- $\mu \mathrm{L}$ of CHCA

135 matrix were deposited on every plate, as positive controls. All the solvents used were MS

136 grade. Mass spectra were obtained on a MicroFlex ${ }^{\mathrm{TM}}$ (Bruker Daltonics) MALDI-TOF MS.

137 Spectra were recorded in the mass range from 2,000 to 20,000 Da.

138 Spectra processing.

139 Spectra were processed using the Biotyper Compass Explorer v4.1 (Bruker Daltonics). Each

140 spectrum was compared with the Mycobacteria bead method Library v.3.0 (Bruker

141 Daltonics). PCA spectra dendrograms were performed using the default parameter set

142 (distance value measured by correlation model with average linkage algorithm, number of

143 clusters maximum defined at four). Dendrogram distances were built by the software on an

144 arbitrary unit based on dissimilarities between spectra, with a range from 0 to 1.2.

145 After the definition of the list of spectra used for the building of the standard cluster, each

146 quadrupled of spectra obtained on the same isolate from the test group were added to the

147 standard list in order to perform a PCA clustering. 81 dendrograms were thus generated, one 
148 for each isolate of the negative and test group. On each dendrogram, the distance separating

149 MALDI-TOF-MS spectra from the tested isolate by spectra from the closest isolate in the 150 reference group was measured. Species from the closest isolate in the reference group were 151 also noted.

\section{RESULTS}

\section{Speciation of mycobacteria based on database match. MALDI-TOF-MS spectra}

155 presenting a low intensity (under 2,000 a.u.) were removed from the study, providing a total 156 number of 411 MALDI-TOF-MS spectra of Mycobacterium species investigated (Tables 1-

157 2), with a mean of 3.91 spectra per isolate. Identification of spectra by comparison with the 158 MALDI Biotyper Compass IVD Library v9.0 and the Mycobacteria bead method Library 159 v.3.0 (Bruker Daltonics) yielded 69\% correct identifications of Mycobacterium species with a 160 comparison score above 2.00; and this value rose to $93 \%$ using a comparison score above 1611.80.

162 Reference cluster. Figure 1 shows the standard PCA dendrogram built with the 90 spectra 163 (Table 1) obtained from M. tuberculosis complex isolates defined as reference groups. One M. tuberculosis isolate was erroneously included in group 3 (M. bovis/ M. africanum) and was thus excluded from the reference cluster. Interestingly, this isolate had been identified by WGS as being a M. tuberculosis lineage 1.1.1 (Indo-Oceanic), the lineage of $M$. tuberculosis most closely related to M. africanum and M. bovis (13). Accordingly, the reference cluster used for further identification of isolates consisted of 23 isolates. The maximum average distance among spectra obtained from replicates of the same bacterial isolate was calculated

170 at $0.420 \pm 0.202$. As shown in Figure 1, the standard cluster presented three main groups here referred to as group 1, 2 and 3. Group 1 presents a top node of separation measured at 0.810 , 
173 obtained on 11 different isolates of M. tuberculosis. Group 2 (top node of separation

174 measured at 0.568 ) presents a coherent clustering with species identification of spectra from

175 five different isolates of M. tuberculosis. Group 3 (top node of separation measured at 0.897)

176 presents clustering of spectra obtained from M. africanum (2), M. bovis (1) and M. bovis

177 BCG isolates (2). No consistent clustering according to M. tuberculosis lineages was

178 observed in clusters 1 and 2 (Figure 2). Reference cluster spectra were deposited on the

179 Méditerranée Infection website (https://www.mediterranee-infection.com/acces-

180 ressources/base-de-donnees/urms-data-base/\#link_acc-25-26-d) and are publicly accessible

181 using doi 10.35081/mbbf-rm88 (https://urldefense.com/v3/ https://www.mediterranee-

182 infection.com/wp-content/uploads/2021/01/Speciation-of-mycobacteria-by-MALDI-TOF-

183 Reference-cluster-dataset.zip_:!JQ5agg!I6GLl-

184 a6ZbO9zQRBdgO1YspBN1OOliEkFyqzKjjwmo0TsExFDQu_FUN5rlC07DPa8jpx\$).

185 Identification of mycobacteria using reference clustering. Observations of 81

186 dendrograms generated with spectra added to the spectra from isolates of the test group are

187 summarised in Table 2. Overall, 77/81 (95.1\%) isolates were correctly identified at the

188 species level (using WGS as the gold standard for identification), including 70/74 (94.6\%)

189 isolates identified in the M. tuberculosis complex and 7/7 isolates identified outside the $M$.

190 tuberculosis complex. In contrast, one M. bovis isolate was misidentified as M. tuberculosis,

191 one M. africanum isolate as M. bovis, and two M. tuberculosis isolates (lineages 2.2.1 and

192 1.1.2) as M. bovis.

193

194 DISCUSSION

195 In this study, we report the unprecedented identification of M. tuberculosis complex species

196 using the MALDI-TOF-MS approach. Data here reported were validated by the fact that

197 positive and negative controls deposited on each MALDI-TOF-MS target plate respectively 
198 correctly identified E. coli (score above 2.00) and no identifications (score under 1.60)

199 against selected database; MALDI-TOF-MS spectra were repeated over at least four repeats;

200 negative controls of the PCA clustering method consisting of 7 isolates of mycobacteria

201 species not included in the M. tuberculosis complex (M. abscessus, M. avium, M. chelonae,

202 M. gilvum, M. persicum) presented spectra with clusterisation distance significantly higher

203 than spectra from isolates of the test groups in the M. tuberculosis complex $(0.892 \pm 0.094$

204 versus $0.398 \pm 0.167 ; \mathrm{p}$ value $=2.39 \mathrm{E}-7$; Student's t-test $)$; all identifications were validated

205 using WGS as the gold standard and spectra from the reference group were deposited in a

206 publicly available database.

207 Here, spectra analysis not only consisted in matching with a database, as reported for

208 most previous studies dealing with MALDI-TOF-MS identification of mycobacteria as a

209 whole and M. tuberculosis complex in particular $(8,9)$, but rather a clustering approach

210 consisting of a PCA against a standard dataset of spectra; similar to the approach previously

211 reported by O'Connor et al. on combined summary spectra (10). Consequently, we are

212 reporting for the first time to the best of our knowledge, the identification of M. tuberculosis

213 complex isolates at the species level, starting from colonies. More precisely, this involves the

214 PCA dendrogram carried out on the 90 spectra of the standard group discriminated $M$.

215 tuberculosis isolates (groups 1 and 2) and non-M. tuberculosis isolates (group 3). Coherent

216 clustering of spectra was observed for 70 isolates among 74 constituting test group (94.6\%),

217 involving a reliable accuracy of clusterisation with the standard cluster among spectra of the

218 same mycobacteria species inside the M. tuberculosis complex. However, of the 74 isolates in

219 the test group, only five clinical isolates were non-tuberculosis mycobacteria, and accuracy of

220 clustering among these five isolates was only $60 \%$. Two inconsistent clusterings consisted in

221 one case of one $M$. africanum isolate that clustered with spectra from $M$. bovis in group 3. As

222 it was observed in group 3 of the standard cluster that spectra from $M$. africanum and $M$. 
223 bovis presented close clustering, this could indicate that the PCA clustering method is not

224 suitable for distinguishing these two species, but does make it possible to distinguish between

225 them and M. tuberculosis. Indeed, O’Connor et al. have already observed that clusterisation

226 of the MALDI-TOF dataset acquired from isolates of the M. tuberculosis complex presented

227 distinct grouping of two on three M. bovis (BCG) isolates compared to other M. tuberculosis

228 isolates, which is coherent with what was observed on the dataset used here (10).

229 Indeed, rapid identification of M. tuberculosis complex isolates at the species level is

230 of medical interest, giving a preliminary information regarding the potential antibiotic

231 susceptibility pattern, as $M$. bovis and M. caprae are intrinsically resistant to pyrazinamide

232 (14) and potential sources of contamination such as M. bovis, M. caprae and M. pinnipedii

233 are three species acknowledged as being responsible for zoonotic tuberculosis (4). In this

234 study however, the lack of consistency of clustering according to M. tuberculosis lineage

235 among groups 1 and 2 indicated that the PCA clustering method reported here did not

236 discriminate the $M$. tuberculosis lineage.

In conclusion, we report on the proof-of-concept that PCA clustering of MALDI-

238 TOF-MS spectra of mycobacteria can be used as a first line method of identification of

239 colonies in the $M$. tuberculosis complex; presenting a reasonable positive predictive value of

240 identification of $94.6 \%$. The method here reported may be refined by increasing the database

241 and may be extended to mycobacteria grown in broth, as all data here presented were

242 acquired from colonies grown on a solid culture medium. The PCA clustering reported here

243 is a more accurate tool for identifying dissimilarities inside groups of closely related spectra

244 than the current identification by comparison with a database. As such, it could be used to

245 investigate ways of discriminating isolates within other bacteria complexes that are still

246 unsolved by MALDI-TOF MS identification. 


\section{ACKNOWLEDGEMENTS.}

249 The authors are very grateful to Gilles Liotaud, Simon Pinchemel and Amaël Fadlane

250 (Assistance Publique-Hôpitaux de Marseille, BSL3 laboratory and Strain Collection of the

251 Rickettsia Unit) for their technical work on mycobacteria culture. MM would like to thank

252 Ghiles Grine for the organisation of genome sequencing. Finally, the authors would like to

253 acknowledge the Head of the Laboratoire National de Référence des IST/VIH et de la

254 Tuberculose, in Niamey, Niger.

255

256 CONFLICT OF INTEREST.

257 MD is a co-founder and shareholder of Culture-Top, a startup whose M4 product is cited in 258 this study.

259

260 FUNDING INFORMATION

261 This work was supported by the French Government under the "Investissements d'avenir"

262 (Investments for the Future) programme managed by the Agence Nationale de la Recherche 263 (ANR, fr: National Agency for Research), (reference: Méditerranée Infection 10-IAHU-03).

264 This work was supported by the Région Provence Alpes Côte d'Azur and European ERDF 265 PRIMI funding.

266 SUPPLEMENTARY MATERIAL

267 The data presented in this study are openly available on the Méditerranée Infection website

268 (https://www.mediterranee-infection.com/acces-ressources/base-de-donnees/urms-data-

269 base/\#link_acc-25-26-d) at [doi: 10.35081/mbbf-rm88].

270 
273 1. Forbes BA, Hall GS, Miller MB, Novak SM, Rowlinson M-C, Salfinger M, Somoskövi

274 A, Warshauer DM, Wilson ML. 2018. Practice Guidelines for Clinical Microbiology

$275 \quad$ Laboratories: Mycobacteria. Clin Microbiol Rev 31.

276 2. Malone KM, Gordon SV. 2017. Mycobacterium tuberculosis Complex Members

277 Adapted to Wild and Domestic Animals. Adv Exp Med Biol 1019:135-154.

278 3. Wanzala SI, Nakavuma J, Travis D, Kia P, Ogwang S, Waters WR, Thacker T, Johnson

279 T, Hadi SA, Sreevatsan S. 2019. Retrospective Analysis of Archived Pyrazinamide

280 Resistant Mycobacterium tuberculosis Complex Isolates from Uganda-Evidence of

281 Interspecies Transmission. Microorganisms 7.

4. Saad J, Baron S, Lagier J-C, Drancourt M, Gautret P. 2020. Mycobacterium bovis

Pulmonary Tuberculosis after Ritual Sheep Sacrifice in Tunisia. Emerg Infect Dis 26:1605-1607.

5. Iketleng T, Lessells R, Dlamini MT, Mogashoa T, Mupfumi L, Moyo S, Gaseitsiwe S, de Oliveira T. 2018. Mycobacterium tuberculosis Next-Generation Whole Genome Sequencing: Opportunities and Challenges. Tuberc Res Treat 2018:1298542. microbiology laboratory: impact of matrix-assisted laser desorption ionization-time of flight mass spectrometry. J Clin Microbiol 51:2182-2194. 
292 7. Lagier J-C, Drancourt M, Charrel R, Bittar F, La Scola B, Ranque S, Raoult D. 2017.

293 Many More Microbes in Humans: Enlarging the Microbiome Repertoire. Clin Infect Dis

$294 \quad 65: S 20-S 29$.

8. Patel R. 2015. MALDI-TOF MS for the diagnosis of infectious diseases. Clin Chem

296

61:100-111.

297

298

9. Alcaide F, Amlerová J, Bou G, Ceyssens PJ, Coll P, Corcoran D, Fangous M-S,

González-Álvarez I, Gorton R, Greub G, Hery-Arnaud G, Hrábak J, Ingebretsen A,

Lucey B, Marekovic I, Mediavilla-Gradolph C, Monté MR, O’Connor J, O’Mahony J, Opota O, O’Reilly B, Orth-Höller D, Oviaño M, Palacios JJ, Palop B, Pranada AB, Quiroga L, Rodríguez-Temporal D, Ruiz-Serrano MJ, Tudó G, Van den Bossche A, van Ingen J, Rodriguez-Sanchez B, European Study Group on Genomics and Molecular Diagnosis (ESGMD). 2018. How to: identify non-tuberculous Mycobacterium species

10. O’Connor JA, Corcoran GD, O’Reilly B, O’Mahony J, Lucey B. 2020. Matrix-Assisted using MALDI-TOF mass spectrometry. Clin Microbiol Infect 24:599-603.

11. Angeletti S, Dicuonzo G, Lo Presti A, Cella E, Crea F, Avola A, Vitali MA, Fagioni M,

310 De Florio L. 2015. MALDI-TOF mass spectrometry and blakpc gene phylogenetic

311 analysis of an outbreak of carbapenem-resistant K. pneumoniae strains. New Microbiol 38:541-550. 
313 12. Zwadyk P, Down JA, Myers N, Dey MS. 1994. Rendering of mycobacteria safe for

314 molecular diagnostic studies and development of a lysis method for strand displacement

315 amplification and PCR. J Clin Microbiol 32:2140-2146.

316 13. Comas I, Coscolla M, Luo T, Borrell S, Holt KE, Kato-Maeda M, Parkhill J, Malla B,

317 Berg S, Thwaites G, Yeboah-Manu D, Bothamley G, Mei J, Wei L, Bentley S, Harris

318 SR, Niemann S, Diel R, Aseffa A, Gao Q, Young D, Gagneux S. 2013. Out-of-Africa

319 migration and Neolithic coexpansion of Mycobacterium tuberculosis with modern

320 humans. Nat Genet 45:1176-1182.

321 14. Feuerriegel S, Köser CU, Richter E, Niemann S. 2013. Mycobacterium canettii is

322 intrinsically resistant to both pyrazinamide and pyrazinoic acid. J Antimicrob

323 Chemother 68:1439-1440. 


\section{Tables: 2}

328 Table 1. Mycobacterial isolates and associated spectra used for building standard PCA

329 clusters

330 Table 2. Observations on individual PCA dendrogram generated on spectra from

331 mycobacteria isolates of the test group

\section{Figures: 2}

333 Figure 1: Standard PCA dendrogram generated with spectra from mycobacteria isolates of the 334 standard group

335 Figure 2: Observation of clusterisation of Mycobacterium tuberculosis lineages among 336 standard PCA dendrograms 
338 Table 1. Mycobacterial isolates and associated spectra used to build standard PCA

339 clusters.

\begin{tabular}{|c|l|l|l|}
\hline Species & Number of isolates & Number of spectra \\
\hline Mycobacterium africanum & 2 & 8 \\
\hline \multirow{2}{*}{$\begin{array}{c}\text { Mycobacterium bovis } \\
\text { Mycobacterium } \\
\text { tuberculosis }\end{array}$} & Lineage 1 (Indo-oceanic) & 3 & 12 \\
\hline Mycobacterium bovis (BCG) & 2 & 8 \\
\hline Lineage 2 (East-Asia) & $3 *$ & $12 *$ \\
\cline { 2 - 5 } & Lineage 3 (East-African-Indian) & 3 & 12 \\
\cline { 2 - 5 } & $\begin{array}{l}\text { Lineage 4 (Euro-American) } \\
\text { TOTAL }\end{array}$ & 8 & 11 \\
\hline
\end{tabular}

340 *: four spectra from one isolate of M. tuberculosis (lineage 1.1.1, Indo-Oceanic) were

341 removed from the reference cluster dataset as it clusterised incorrectly. 
343 Table 2. Observations on an individual PCA dendrogram generated on spectra from

344 mycobacteria isolates of the test group.

\begin{tabular}{|l|l|l|l|l|l|}
\hline Isolates species & $\begin{array}{l}\text { Number of } \\
\text { isolates }\end{array}$ & $\begin{array}{l}\text { Number of } \\
\text { spectra }\end{array}$ & $\begin{array}{l}\text { Mean } \\
\text { distance of } \\
\text { clusterisation }\end{array}$ & $\begin{array}{l}\text { Standard } \\
\text { deviation of } \\
\text { distance of } \\
\text { clusterisation }\end{array}$ & $\begin{array}{l}\text { Consistency of } \\
\text { clusterisation } \\
\text { with closest } \\
\text { species }\end{array}$ \\
\hline
\end{tabular}

Negative control:

\begin{tabular}{|l|l|l|l|l|l|}
\hline Mycobacterium abscessus & 2 & 8 & 0.827 & NA & NA \\
\hline Mycobacterium avium & 2 & 8 & 0.938 & NA & NA \\
\hline Mycobacterium chelonae & 1 & 4 & 0.928 & NA & NA \\
\hline Mycobacterium gilvum & 1 & 4 & 0.995 & NA & NA \\
\hline Mycobacterium persicum & 1 & 4 & 0.795 & NA & NA \\
\hline TOTAL Negative controls & 7 & 28 & 0.892 & 0.094 & NA \\
\hline
\end{tabular}

Test group:

\begin{tabular}{|l|l|l|l|l|l|}
\hline Mycobacterium africanum & 1 & 4 & 0.333 & NA & $0 \%$ \\
\hline Mycobacterium bovis & 3 & 12 & 0.458 & 0.067 & $66.7 \%$ \\
\hline Mycobacterium bovis $($ BCG) & 1 & 4 & 0.750 & NA & $100 \%$ \\
\hline Mycobacterium tuberculosis & 69 & 269 & 0.387 & 0.167 & $97.1 \%$ \\
\hline TOTAL Test group & 74 & 289 & 0.398 & 0.167 & $94.6 \%$ \\
\hline
\end{tabular}




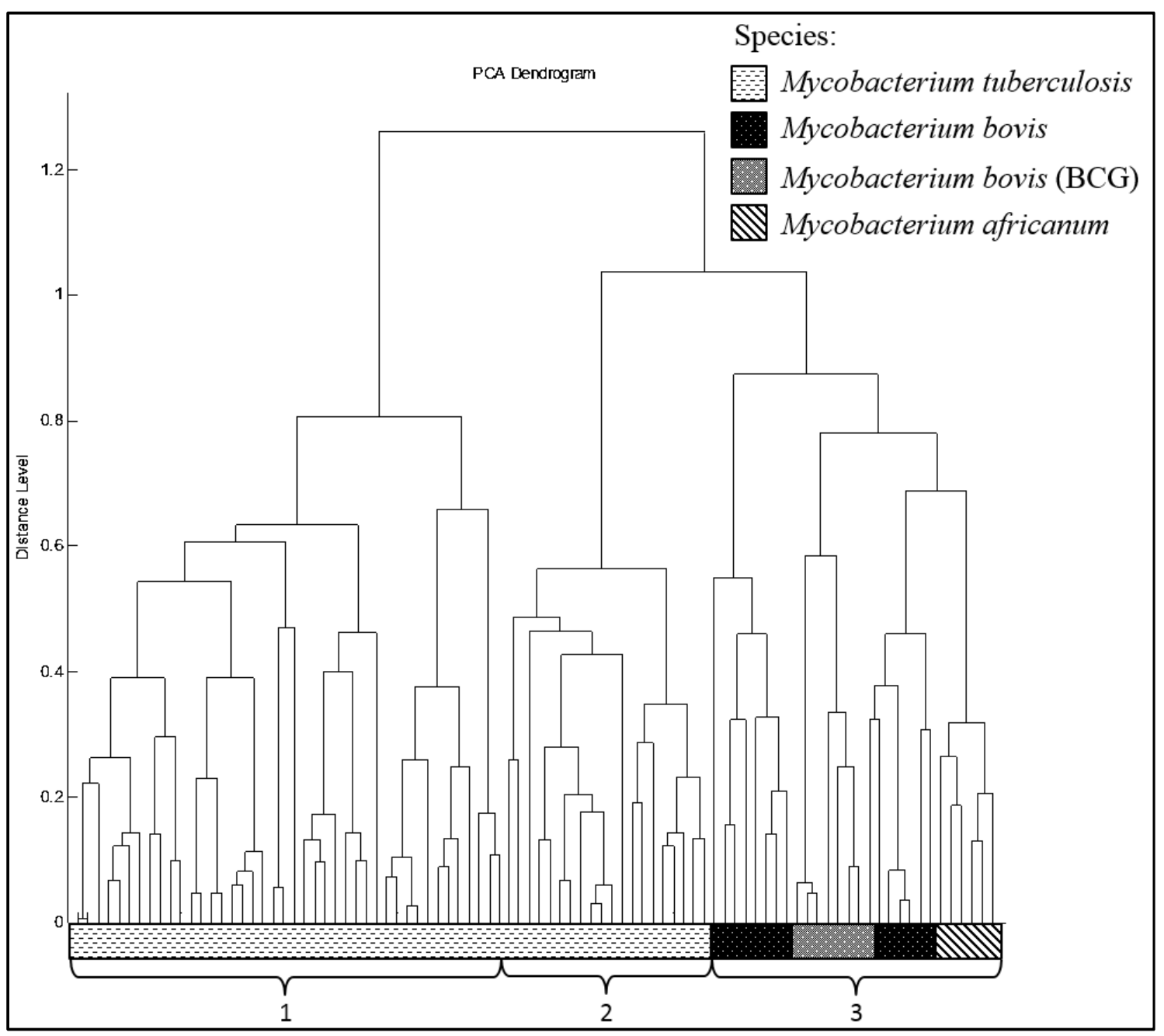

348 Figure 1. Standard PCA dendrogram generated with spectra from mycobacteria

349 isolates in the standard group. 


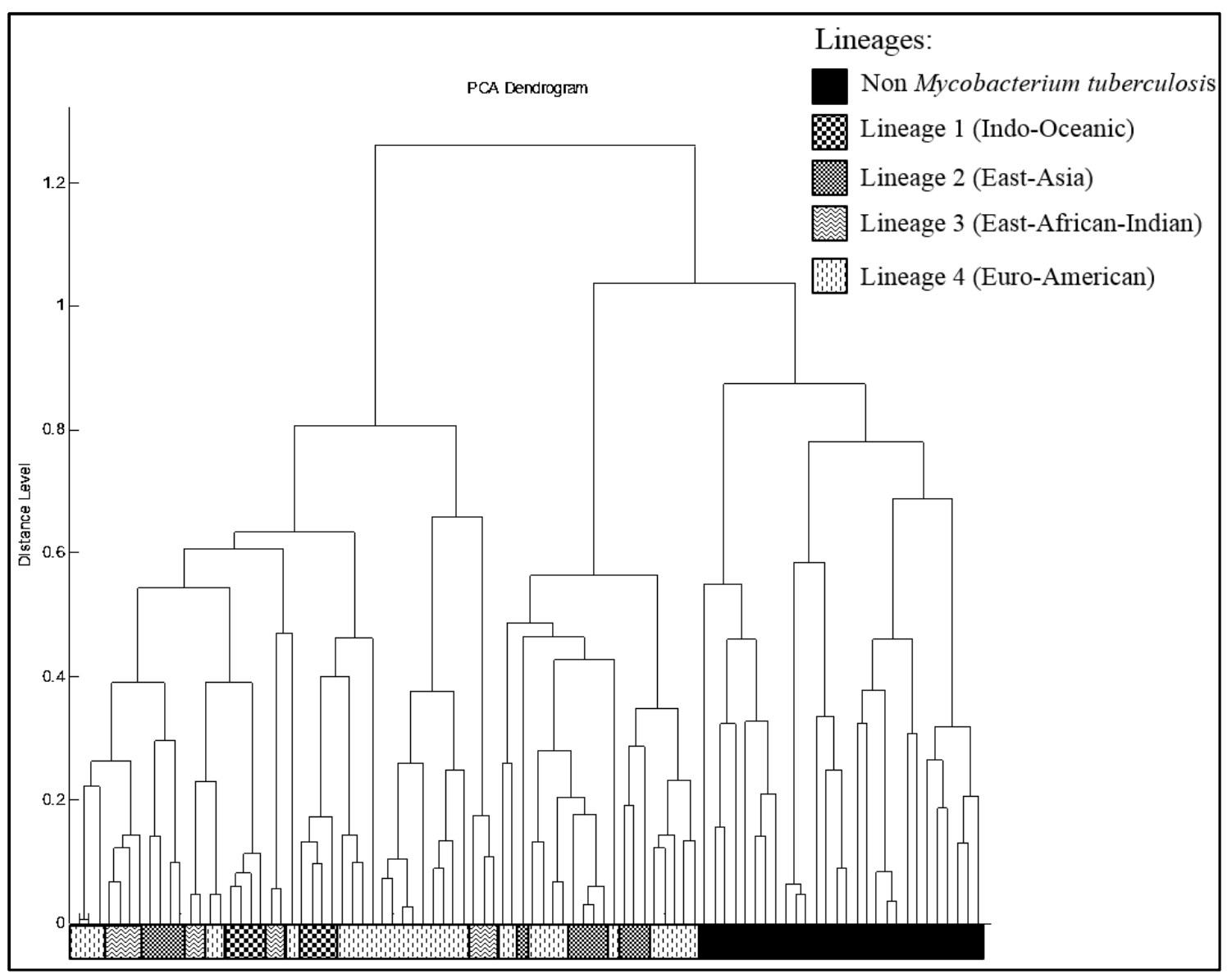

352 Figure 2: Observation of clusterisation of Mycobacterium tuberculosis lineages among 


\section{Species:}

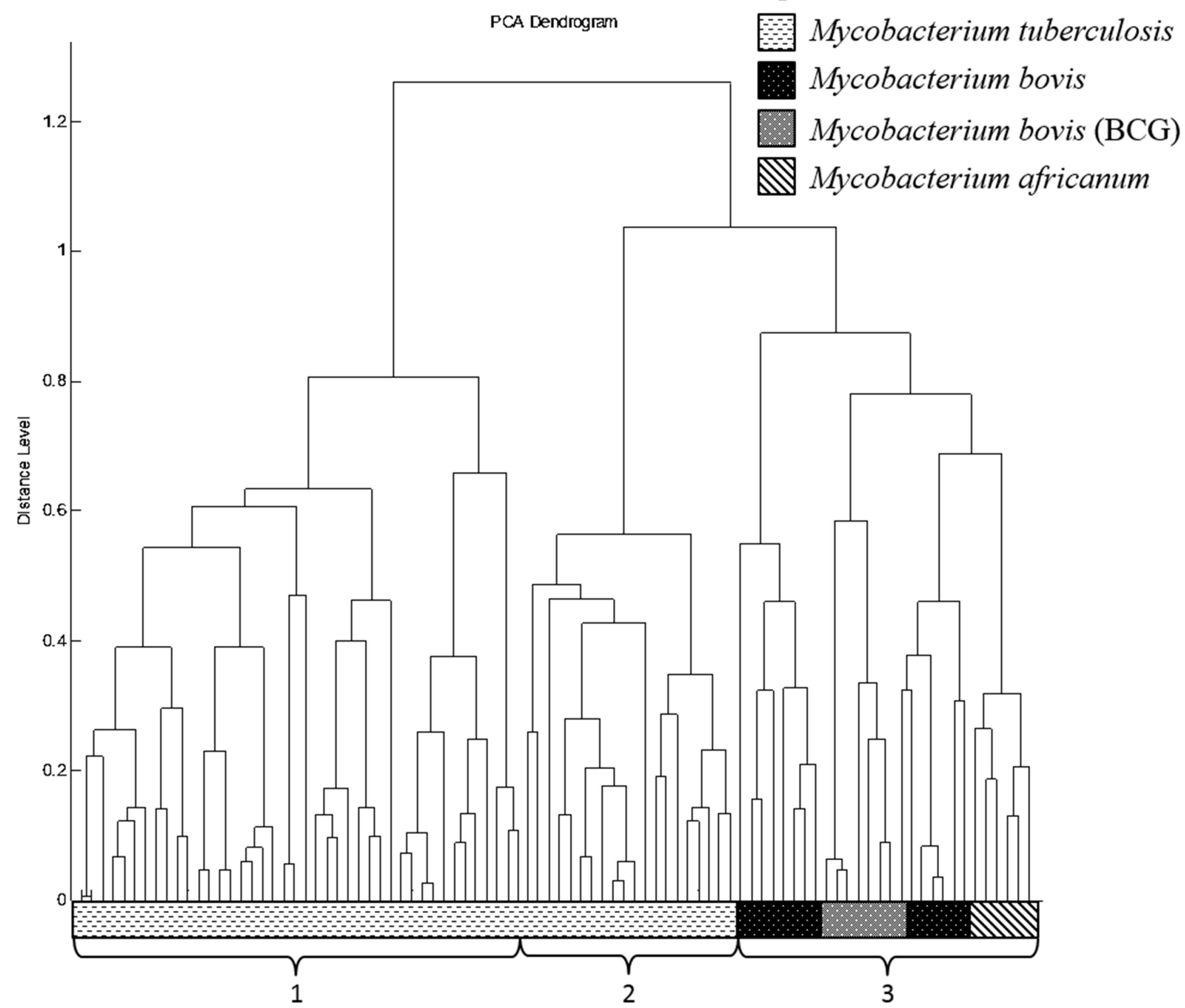


Lineages:

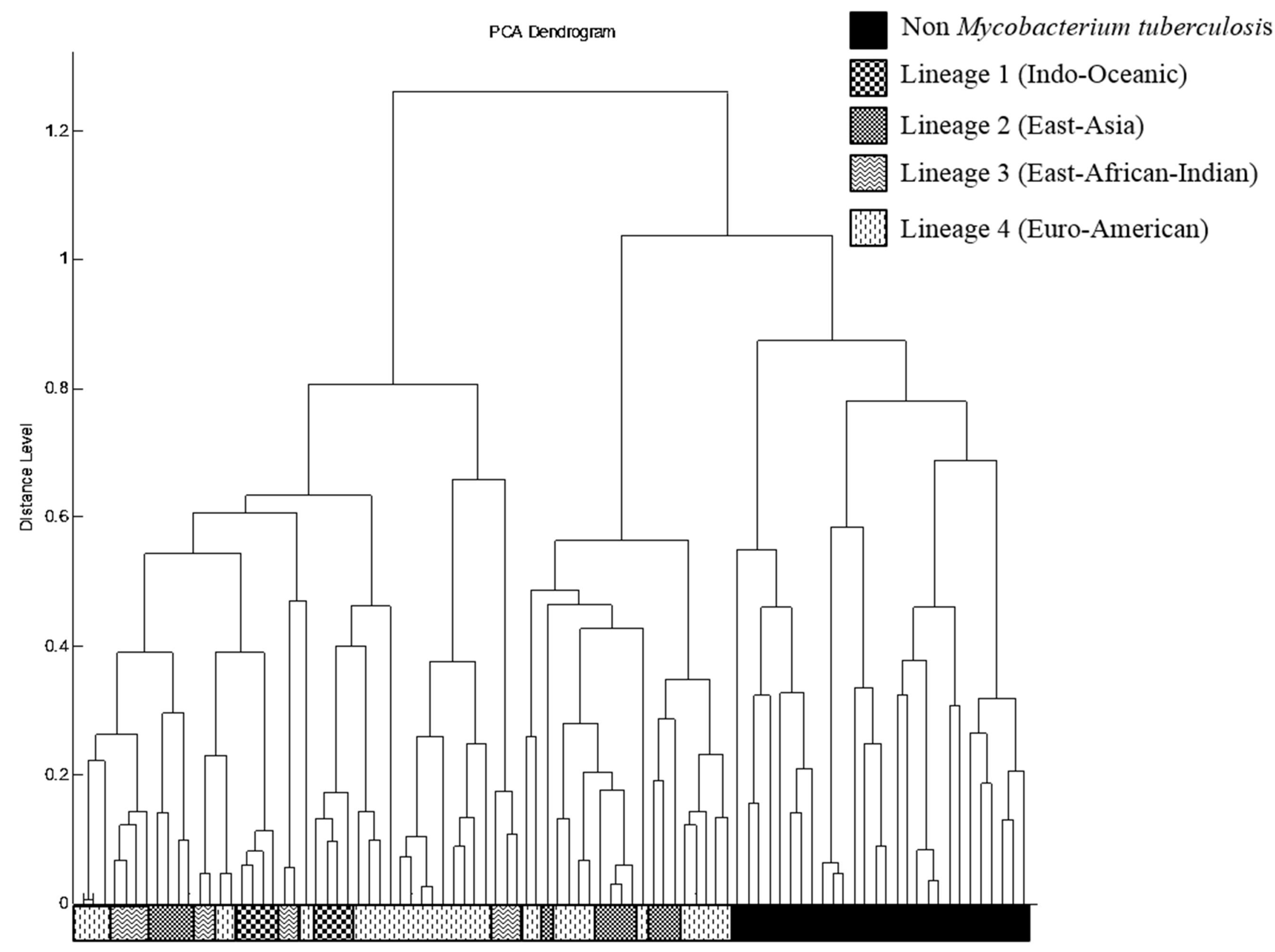

